University of South Carolina

Scholar Commons

$12-15-2003$

\title{
Accumulation of Electron Spin Polarization at Semiconductor Interfaces
}

Yuriy V. Pershin Dr

University of South Carolina - Columbia, pershin@physics.sc.edu

Follow this and additional works at: https://scholarcommons.sc.edu/phys_facpub

Publication Info

Published in Physical Review B, ed. Gene D. Sprouse, Volume 68, Issue 23, 2003, pages

233309-1-233309-4.

Pershin, Y. V. (2003). Accumulation of electron spin polarization at semiconductor interfaces. Physical Review B, 68(23), 233309-1 - 233309-4. DOI: 10.1103/PhysRevB.68.233309

(c) Physical Review B, 2003, American Physical Society

This Article is brought to you by the Physics and Astronomy, Department of at Scholar Commons. It has been accepted for inclusion in Faculty Publications by an authorized administrator of Scholar Commons. For more information, please contact digres@mailbox.sc.edu. 


\title{
Accumulation of electron spin polarization at semiconductor interfaces
}

\author{
Yu. V. Pershin \\ Center for Quantum Device Technology, Clarkson University, Potsdam, New York 13699-5720, USA
}

(Received 17 September 2003; published 31 December 2003)

\begin{abstract}
In this Brief Report we study theoretically the propagation of electron spin polarization through an interface separating two $n$-type semiconductor regions within the two-component drift-diffusion model in an applied electric field. It is assumed that inhomogeneous spin polarization is created locally by a continuous source of spin polarization and is driven through the boundary by the electric field. The spin polarization distribution is calculated analytically. We find that for specific values of parameters describing the system, the electron spin polarization is accumulated near the interface. A simple analytical expression for the amplitude of spin accumulation as a function of the system parameters is found. The obtained results will be useful in designing new spintronic devices.
\end{abstract}

DOI: 10.1103/PhysRevB.68.233309

PACS number(s): 72.25.Dc, 72.20.Ht, 72.25.Hg, 72.25.Mk

\section{INTRODUCTION}

Numerous proposals for solid-state devices based on the manipulation of electron ${ }^{1-10}$ and nuclear spins ${ }^{11-14}$ have given a renewed impetus to theoretical and experimental investigations of spin-related effects in semiconductors. ${ }^{15-20}$ Operation of a semiconductor spintronic device, analogous, for example, to a Datta-Das transistor, ${ }^{1}$ requires efficient spin injection into a semiconductor, spin manipulation, control and transport, and also spin detection. Once injected into a spintronic device, electrons experience spin-dependent interactions with the environment, which cause relaxation. It is of value to understand the mechanisms of electron spin relaxation and find the ways to increase electron spin polarization density.

In our recent paper $^{2}$ we have proposed to use an inhomogeneous doping of semiconductors to compress and amplify the spin polarization density. It was shown numerically that a broad pulse of spin polarization can be squeezed and amplified near a boundary separating two differently doped semiconductor regions. The built-in electric field at the boundary accelerates the propagation of the spin polarization through the boundary, if the spin polarization passes from the lowdoped region to high-doped region. Spin amplification occurs past the boundary, within the distance of the order of the depletion layer width. In the present paper we extend our previous research by characterizing the semiconductor regions by two additional parameters besides the doping density: the electron diffusion coefficient and the electron spin relaxation time. The extended set of parameters allows us to describe not only a semiconductor monojunction, but also a semiconductor hetero-junction as well. Neglecting the effect of charge accumulation/redistribution near the interface, we analytically find the electron spin polarization distribution created by a continuous source of spin polarization. The main result of our research, Eq. (10) below, expresses the spin accumulation amplitude at the interface as a function of the system parameters. Moreover, in the present Brief Report we compare our analytical results with results obtained numerically.

The geometry of the system under investigation is depicted in Fig. 1. Continuous source of spin polarization is located at $x=x_{0}$ in the left semiconductor region with the doping density $N_{1}$, the electron diffusion coefficient $D_{1}$, and the electron spin relaxation time $\tau_{s f}^{1}$. The right semiconductor region is characterized by the following set of parameters: $N_{2}, D_{2}$, and $\tau_{s f}^{2}$, respectively. Under the influence of the applied electric field, the spin polarized carriers drift through the interface located at $x=0$ into the right semiconductor region. We are not specifying the length of these regions, selecting the current as the external control parameter rather than the applied voltage. Moreover, the structure is assumed to be sufficiently thick in transverse directions to allow for one-dimensional electrostatic treatment. In what follows we calculate the distribution of the spin polarization density in such a system.

From the experimental standpoint, non-equilibrium spinpolarization can be created locally in the bulk of a semiconductor, for example, by using ferromagnetic-metal scanning tunneling microscopy tips, ${ }^{21,22}$ or by optical pumping techniques. ${ }^{23-25}$ Alternative spin polarization mechanisms are also possible. ${ }^{26-30}$ In the following sections, we study the propagation of non-equilibrium spin polarization through the boundary between the low-doped and high-doped regions.

\section{MODEL}

Our theoretical investigation is based on the twocomponent drift-diffusion model (see, e.g., Refs. 31 and 32).

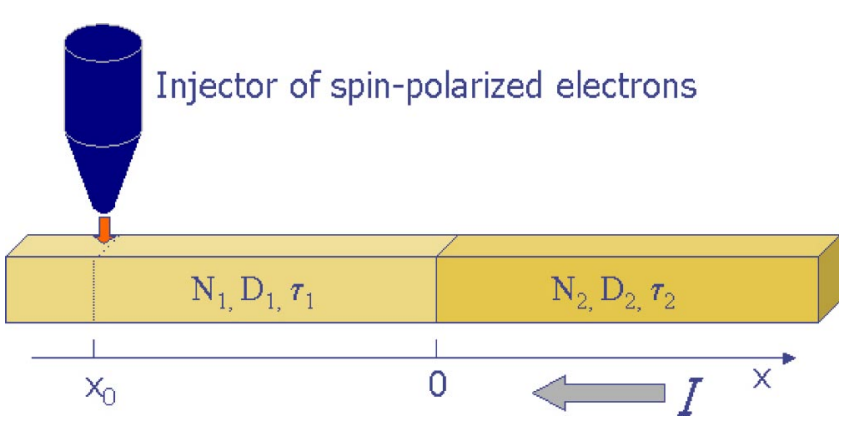

FIG. 1. (Color online) Schematic representation of the system under investigation: spin-polarized electrons are injected at $x=x_{0}$ and move toward the interface located at $x=0$ under the action of the electric field. 
In our case, the system is described by the following set of equations:

$$
\begin{gathered}
e \frac{\partial n_{\uparrow(\downarrow)}^{i}}{\partial t}=\operatorname{div} \vec{j}_{\uparrow(\downarrow)}^{i}+\frac{e}{2 \tau_{s f}^{i}}\left(n_{\downarrow(\uparrow)}^{i}-n_{\uparrow(\downarrow)}^{i}\right)+S_{\uparrow(\downarrow)}(\vec{r}, t), \\
\vec{j}_{\uparrow(\downarrow)}^{i}=\sigma_{\uparrow(\downarrow)}^{i} \vec{E}_{i}+e D_{i} \nabla n_{\uparrow(\downarrow)}^{i},
\end{gathered}
$$

and

$$
\sigma_{\uparrow(\downarrow)}^{i}=e n_{\uparrow(\downarrow)}^{i} \mu_{i},
$$

where $e$ is the electron charge, $n_{\uparrow(\downarrow)}^{i}$ is the density of spin-up (spin-down) electrons, the index $i=1$ (2) identifies the left (right) semiconductor region, $\vec{j}_{\uparrow(\downarrow)}^{i}$ is the current density, $S_{\uparrow(\downarrow)}(\vec{r}, t)$ describes the source of the spin polarization, $\sigma_{\uparrow(\downarrow)}^{i}$ is the conductivity, and $\mu_{i}$ is the mobility, connected with the diffusion coefficient $D_{i}$ via the Einstein relation $\mu_{i}$ $=D_{i} e /\left(k_{B} T\right)$, and defined via $\vec{v}_{d r i f t}^{i}=\mu_{i} \vec{E}_{i}$.

Equation (1) is the usual continuity relation that takes into account spin relaxation and the source of the spin polarization, Eq. (2) is the expression for the current which includes the drift and diffusion contributions, and Eq. (3) is the expression for the conductivity. It is assumed that the diffusion coefficient $D_{i}$ and the spin relaxation time $\tau_{s f}^{i}$ are equal for spin-up and spin-down electrons in the same semiconductor region.

To separate the equations for the charge and spin degrees of freedom, we introduce the charge density $n_{i}=n_{\uparrow}^{i}+n_{\downarrow}^{i}$ and the spin polarization density $P_{i}=n_{\uparrow}^{i}-n_{\downarrow}^{i}$. We assume that at room temperature the density of the ionized donors $N_{i}$ is equal to the donor density $\left(N_{i}=N_{1}\right.$ for $x<0$ and $N_{i}=N_{2}$ for $x>0$ ), i.e., all the donors are ionized. Moreover, we neglect the effect of the electron density redistribution near the interface. We had already studied this effect numerically; ${ }^{2}$ the comparison of the numerical solution with analytical solution (reported here) will be given below. Under such assumptions, the electron densities at both semiconductors are equal to the donor densities.

In our calculations, we use the current $j_{0}$ through the sample as the external control parameter. The current as the external control parameter, rather than the applied voltage, is more convenient because the current is constant throughout the electric circuit that contains the sample. If we use the voltage as the external control parameter, we have to take into account voltage drops in different parts of the circuit, such as, for example, at the Schottky barriers between metal and semiconductor. Since we assume that the electron charge density is homogeneous in each semiconductor region, the current does not have the diffusion component and the electric field is simply connected to the current: $E_{i}=j_{0} / \sigma_{i}$.

From the set of relations (1)-(3), we obtain an equation for the spin polarization density:

$$
\frac{\partial P_{i}}{\partial t}=D_{i} \Delta P_{i}+D_{i} \frac{e \vec{E}_{i}}{k_{B} T} \nabla P_{i}-\frac{P_{i}}{\tau_{s f}^{i}}+F(\vec{r}, t) .
$$

Here $F(\vec{r}, t)=\left[S_{\uparrow}(\vec{r}, t)-S_{\downarrow}(\vec{r}, t)\right] / e$ represents the polarization density created by the external source. For the experi- mental situation shown in Fig. 1 the function describing the source of spin polarization density is selected in the following form: $F(\vec{r}, t)=F_{0} \delta\left(x-x_{0}\right)$, where the constant $F_{0}$ measures the spin polarization density created per unit time.

\section{RESULTS AND DISCUSSION}

To make our calculations more transparent, we rewrite Eq. (4) explicitly for the left and right semiconductor regions:

$$
\begin{gathered}
D_{1} \Delta P_{1}+D_{1} \frac{e E_{1}}{k_{B} T} \nabla P_{1}-\frac{P_{1}}{\tau_{s f}^{1}}+F_{0} \delta\left(x-x_{0}\right)=0, \quad x<0, \\
D_{2} \Delta P_{2}+D_{2} \frac{e E_{2}}{k_{B} T} \nabla P_{2}-\frac{P_{2}}{\tau_{s f}^{2}}=0, \quad x>0 .
\end{gathered}
$$

The boundary conditions are imposed by the following requirements: the current conservation at the boundary and the continuity of spin polarization density at the boundary. They are

$$
\begin{aligned}
\mu_{1} E_{1} P_{1}(0)+D_{1} \nabla P_{1}(0) & =\mu_{2} E_{2} P_{2}(0)+D_{2} \nabla P_{2}(0), \\
P_{1}(0) & =P_{2}(0),
\end{aligned}
$$

Solution of Eqs. (5) with boundary conditions (6) can be written in forms

$$
\begin{gathered}
P_{1}(x)=P_{\delta}\left(x-x_{0}\right)+B e^{\alpha_{1} x}, \\
P_{2}(x)=C e^{\alpha_{2} x},
\end{gathered}
$$

where $P_{\delta}(x)$ is a particular solution of Eq. (5a), given below, and $\alpha_{i}$ are the roots of the quadratic equation $\alpha_{i}^{2}$ $+\left(e E_{i} / k_{B} T\right) \alpha_{i}-1 /\left(D_{i} \tau_{i}\right)=0, \quad i=1,2$. The choice of the roots is defined by the boundary conditions at $x= \pm \infty$ : $\alpha_{1}$ must be positive and $\alpha_{2}$ must be negative. The function $P_{\delta}(x)$ can be easily found using the Fourier transform of Eq. (5a); it has the following form:

$$
P_{\delta}(x)=F_{0} \sqrt{\frac{\tau_{s f}^{1}}{D_{1}}} \frac{e^{-\frac{x}{2 \sqrt{D_{1} \tau_{s f}^{1}}}\left(A_{1}+\operatorname{sgn}(x) \sqrt{A_{1}^{2}+4}\right)}}{\sqrt{A_{1}^{2}+4}},
$$

with $A_{1}=\left(e E_{1} / k_{B} T\right) \sqrt{D_{1} \tau_{s f}^{1}}$. The function $P_{\delta}(x)$ gives the spin polarization density distribution created by the point source for an homogeneous semiconductor. Equation (8) shows that in the absence of electric field $\left(A_{1}=0\right)$ the spin polarization density distribution is symmetrical around $x$ $=0$. Non-zero electric field leads to asymmetry of spin polarization density, which exponentially decays on the length scales of

$$
L_{1,2}=\frac{2 \sqrt{D_{i} \tau_{s f}^{i}}}{\left(A_{1} \pm \sqrt{A_{1}^{2}+4}\right)},
$$




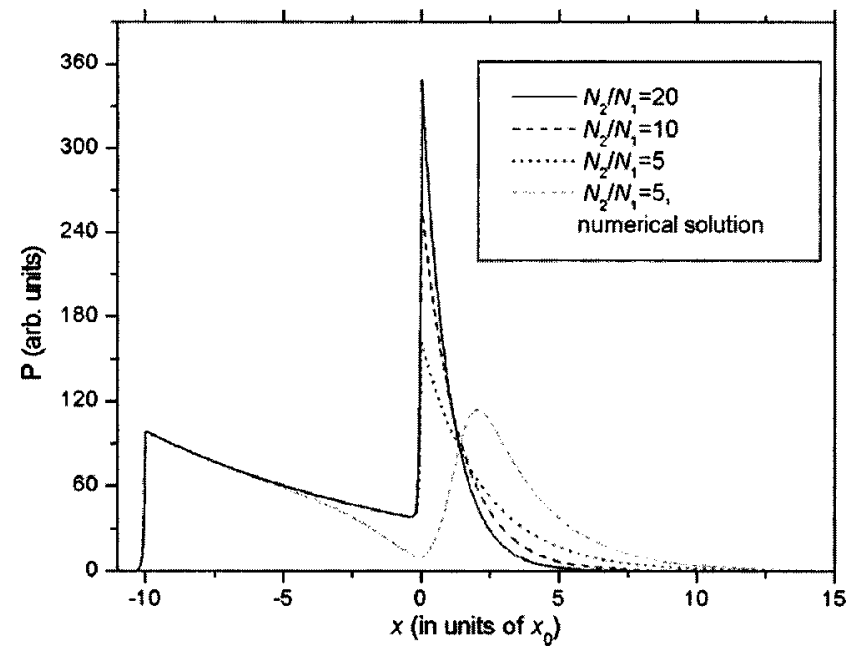

FIG. 2. Spin polarization density created by a point source at $x=-10$ as a function of $x$ for different doping densities $N_{2}$ and for $\tau_{1}=\tau_{2}$ and $D_{1}=D_{2}$, showing an increase of the spin accumulation with higher $N_{2}$. The dash-dotted line represents the spin polarization density obtained with taking into account the redistribution of the electron density near the boundary (see Fig. 4 from Ref. 2).

called the down-stream and up-stream spin diffusion lengths. ${ }^{31}$ Another consequence of the electric field is a decrease of spin polarization density at $x=0$ as $1 / \sqrt{A_{1}^{2}+4}$.

Using boundary conditions (7), we calculate the coefficients $B$ and $C$ :

$$
\begin{gathered}
B=C-P_{\delta}\left(-x_{0}\right) \\
C=\frac{\alpha_{1} P_{\delta}\left(-x_{0}\right)-P_{\delta}^{\prime}\left(-x_{0}\right)}{\left(\frac{e E_{1}}{k_{B} T}+\alpha_{1}\right)-\frac{D_{2}}{D_{1}}\left(\frac{e E_{2}}{k_{B} T}+\alpha_{2}\right)} .
\end{gathered}
$$

Figure 2 represents the spin polarization density as a function of the coordinate. In order to compare the analytical results with numerical results reported in Ref. 2, Fig. 2 was drawn for $\tau_{s f}^{1}=\tau_{s f}^{2}$ and $D_{1}=D_{2}$. We have obtained a good agreement between analytical and numerical results. The dependence of the peak value of the spin polarization density on the doping density is similar: the peak value of the spin polarization density increases as the doping density of the second semiconductor region increases. The peak value of the spin polarization density calculated analytically (without taking into account the effect of charge redistribution/ accumulation at the interface) is of the order of $20 \%$ higher in comparison with the peak value of the spin polarization density calculated numerically. Moreover, the effect of charge redistribution/accumulation at the interface results in the shift of the peak value of the spin polarization density from the interface for the distance of the order of the depletion layer width. ${ }^{2}$

Let us consider in detail the coefficient $C$ given by expression (9b). After a straightforward algebra, it can be rewritten in the following form:

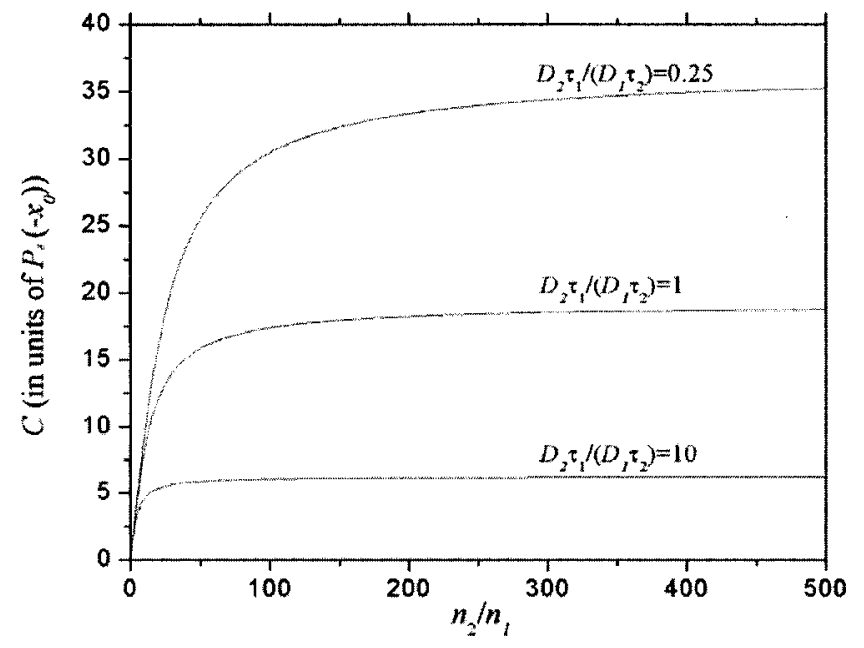

FIG. 3. The amplitude of the spin accumulation $C$ as a function of $n_{2} / n_{1}$ for different values of $D_{2} \tau_{1} / D_{1} \tau_{2}$ and for $A_{1}=20$.

$$
\begin{aligned}
C= & \frac{2 \sqrt{A_{1}^{2}+4}}{\sqrt{A_{1}^{2}+4}+\sqrt{A_{1}^{2}\left(\frac{n_{1}}{n_{2}}\right)^{2}+4 \frac{D_{2} \tau_{1}}{D_{1} \tau_{2}}}+A_{1}-A_{1} \frac{n_{1}}{n_{2}}} \\
& \times P_{\delta}\left(-x_{0}\right) .
\end{aligned}
$$

It can be readily seen that $P_{\delta}\left(-x_{0}\right)$ in Eq. (10) is the value of $C$ when two semiconductor regions have the same properties. Consequently, the factor before $P_{\delta}\left(-x_{0}\right)$ in Eq. (10) gives the change of the spin polarization density at the boundary due to the semiconductor mismatch. Since the numerator of the fraction in expression (10) is defined only by the properties of the first semiconductor, the maximum value of $C$ corresponds to minimum of the denominator in Eq. (10). For the experimental situation depicted on Fig. 1, the parameter $A_{1}$ is negative. Then, the peak value of the spin polarization density is maximal when

$$
\frac{n_{1}}{n_{2}} \ll 1 \quad \text { and } \quad \frac{D_{2} \tau_{1}}{D_{1} \tau_{2}} \ll 1
$$

If conditions (11) are satisfied, the constant $C$ takes the form

$$
C=2 P_{\delta}\left(-x_{0}\right) /\left(1-\frac{\left|A_{1}\right|}{\sqrt{A_{1}^{2}+4}}\right)
$$

Moreover, if $\left|A_{1}\right| \gg 1$, then $C=A_{1}^{2} P_{\delta}\left(-x_{0}\right)$.

The peak value of the spin polarization density as a function of the doping level of the second semiconductor at selected values of the parameter $D_{2} \tau_{1} /\left(D_{1} \tau_{2}\right)$ is shown in Fig. 3 . The peak value of the spin polarization density increases with a decrease of $D_{2} \tau_{1} /\left(D_{1} \tau_{2}\right)$ and with an increase of $n_{2}$. It is interesting to note that the sharp growth of the peak value of the spin polarization occurs at relatively small values of parameter $n_{2} / n_{1}$ (in Fig. 3 for $n_{2} / n_{1} \leqslant 100$ ). The subsequent growth of this parameter results in a relatively small increase of the peak value of the spin polarization density. 


\section{CONCLUSIONS}

In conclusion, the propagation of spin-polarized electrons through an interface separating two different semiconductor regions was investigated analytically. We have obtained an explicit expression for the peak value of the spin polarization density in terms of the system parameters. To obtain a high level of the spin polarization density at the interface, it is necessary that both semiconductors have long electron spin relaxation times; the right semiconductor should have a small electron diffusion coefficient and a high doping level. The analytical results are in good agreement with results obtained numerically earlier. The formula for the peak value of the spin polarization density could be useful in engineering of spintronic devices.

\section{ACKNOWLEDGMENTS}

We gratefully acknowledge helpful discussions with Professor M.-C. Cheng, Professor V. N. Gorshkov, Professor V. Privman, and Professor I. D. Vagner. This research was supported in part by the National Security Agency and Advanced Research and Development Activity under Army Research Office Contract No. DAAD-19-02-1-0035, and by the National Science Foundation, Grant No. DMR-0121146.
${ }^{1}$ S. Datta and B. Das, Appl. Phys. Lett. 56, 665 (1990).

${ }^{2}$ Yu. V. Pershin and V. Privman, Phys. Rev. Lett. 90, 256602 (2003).

${ }^{3}$ M. Johnson, Science 260, 320 (1993).

${ }^{4}$ M. E. Flatte and G. Vignale, Appl. Phys. Lett. 78, 1273 (2001).

${ }^{5}$ I. Zutic, J. Fabian, and S. Das Sarma, Appl. Phys. Lett. 79, 1558 (2001).

${ }^{6}$ C. Ciuti, J. P. McGuire, and L. J. Sham, Appl. Phys. Lett. 81, 4781 (2002).

${ }^{7}$ T. Koga, J. Nitta, H. Takayanagi, and S. Datta, Phys. Rev. Lett. 88, 126601 (2002)

${ }^{8}$ X. F. Wang, P. Vasilopoulos, and F. M. Peeters, Phys. Rev. B 65, 165217 (2002).

${ }^{9}$ J. Schliemann, J. C. Egues, and D. Loss, Phys. Rev. Lett. 90, 146801 (2003).

${ }^{10}$ J. Fabian, I. Zutic, and S. Das Sarma, Phys. Rev. B 66, 165301 (2002).

${ }^{11}$ R. G. Mani, W. B. Johnson, V. Narayanamurti, V. Privman, and Y.-H. Zhang, Physica E (Amsterdam) 12, 152 (2002).

${ }^{12}$ Yu. V. Pershin, S. N. Shevchenko, I. D. Vagner, and P. Wyder, Phys. Rev. B 66, 035303 (2002).

${ }^{13}$ Yu. V. Pershin, I. D. Vagner, and P. Wyder, J. Phys.: Condens. Matter 15, 997 (2003).

${ }^{14}$ B. E. Kane, L. N. Pfeiffer, and K. W. West, Phys. Rev. B 46, 7264 (1992).

${ }^{15}$ G. Prinz, Science 282, 1660 (1998).

${ }^{16}$ S. A. Wolf, D. D. Awschalom, R. A. Buhrman, J. M. Daughton, S. von Molnár, M. L. Roukes, A. Y. Chtchelkanova, and D. M. Treger, Science 294, 1488 (2001).
${ }^{17}$ S. Das Sarma, J. Fabian, X. Hu, and I. Zutic, IEEE Trans. Magn. 36, 2821 (2000); S. Das Sarma, Am. Sci. 89, 516 (2001).

${ }^{18}$ Yu. V. Pershin and V. Privman, cond-mat/0309513, Phys. Rev. B (to be published); Yu. V. Pershin, cond-mat/0310225 (unpublished).

${ }^{19}$ D. D. Awschalom, M. E. Flatte, and N. Samarth, Sci. Am. 286, 66 (2002).

${ }^{20}$ S. Saikin, M. Shen, M.-C. Cheng, and V. Privman, J. Appl. Phys. 94, 1769 (2003).

${ }^{21}$ S. F. Alvarado and P. Renaud, Phys. Rev. Lett. 68, 1387 (1992).

${ }^{22}$ S. F. Alvarado, Phys. Rev. Lett. 75, 513 (1995).

${ }^{23}$ J. M. Kikkawa and D. D. Awschalom, Phys. Rev. Lett. 80, 4313 (1998).

${ }^{24}$ J. M. Kikkawa and D. D. Awschalom, Nature (London) 397, 139 (1999).

${ }^{25}$ D. Hägele, M. Oestreich, W. W. Rühle, N. Nestle, and K. Eberl, Appl. Phys. Lett. 73, 1580 (1998).

${ }^{26}$ J. Nitta, T. Akazaki, H. Takayanagi, and T. Enoki, Phys. Rev. Lett. 78, 1335 (1997).

${ }^{27}$ G. Meier, T. Matsuyama, and U. Merkt, Phys. Rev. B 65, 125327 (2002).

${ }^{28}$ Yu. V. Pershin and V. Privman, Nano Lett. 3, 695 (2003).

${ }^{29}$ K. R. Wald, L. P. Kouwenhoven, P. L. McEuen, N. C. van der Vaart, and C. T. Foxon, Phys. Rev. Lett. 73, 1011 (1994).

${ }^{30}$ D. C. Dixon, K. R. Wald, P. L. McEuen, and M. R. Melloch, Phys. Rev. B 56, 4743 (1997).

${ }^{31}$ Z. G. Yu and M. E. Flatté, Phys. Rev. B 66, 235302 (2002).

${ }^{32}$ Z. G. Yu and M. E. Flatté, Phys. Rev. B 66, 201202 (2002). 